\title{
Comparison of CCD astrolabe multi-site solar diameter observations
}

\author{
A. H. Andrei ${ }^{1}$, S. C. Boscardin ${ }^{2}$, F. Chollet ${ }^{3,4}$, C. Delmas ${ }^{5}$, O. Golbasi ${ }^{3,6}$, E. G. Jilinskii ${ }^{7,8}$, H. Kiliç ${ }^{6}$, F. Laclare ${ }^{5}$, \\ F. Morand ${ }^{5}$, J. L. Penna ${ }^{7}$, and E. Reis Neto ${ }^{7}$ \\ ${ }^{1}$ GEA - Observatório Nacional/MCT e Observatório do Valongo/UFRJ, Ladeira Pedro Antônio 43, Rio de Janeiro, Brasil \\ e-mail: oat1@ov.ufrj.br \\ 2 Observatório do Valongo/UFRJ, Brasil \\ 3 Tubitak National Observatory, Turkey \\ ${ }^{4}$ Observatoire de Paris, France \\ 5 Dptm. GEMINI UMR 6203-Observatoire de la Côte d'Azur, France \\ ${ }^{6}$ Akdeniz University, Turkey \\ 7 Observatório Nacional/MCT, Brasil \\ ${ }^{8}$ Pulkovo Observatory, Russia
}

Received 21 May 2004 / Accepted 5 July 2004

\begin{abstract}
Results are presented of measured variations of the photospheric solar diameter, as concurrently observed at three sites of the R2S3 (Réseau de Suivi au Sol du Rayon Solaire) consortium in 2001. Important solar flux variations appeared in that year, just after the maximum of solar activity cycle 23, make that time stretch particularly promising for a comparison of the multi-site results. The sites are those in Turkey, France and Brasil. All observations are made with similar CCD solar astrolabes, and at nearby effective wavelengths. The data reductions share algorithms, that are alike, the outcomes of which are here treated after applying a normalization correction using the Fried parameter. Since the sites are geographically quite far, atmospheric conditions are dismissed as possible causes of the large common trend found.

Owing to particularities of each site, the common continuous observational period extends from April to September. The standard deviation for the daily averages is close to 0.'47 for the three sites. Accordingly, the three series are smoothed by a low-pass-band Fourier filter of 150 observations (typically one month). The main common features found are a declining linear trend, of the order of 0.7 mas/day, and a relative maximum, around MJD 2120, of the order of 100 mas. Standard statistical tests endorse the correlation of the three series.
\end{abstract}

Key words. Sun: general - Sun: fundamental parameters - Sun: photosphere - Sun: activity

\section{Introduction}

Variations of the measured solar radius, on the scale of several hundredths of arc second, have now been repeatedly reported by several authors (Laclare et al. 1996; Wittmann 1997; Noel 2004; Chollet \& Sinceac 1999; Emilio et al. 2000; Reis Neto et al. 2003), either using similar or entirely different instruments. In spite of this there are weigthy questions to be answered, like the precise description of the mechanism triggering such variations, and the extent of noise (or even modulation) on ground-based observations caused by atmospheric disturbances (Lakhal et al. 1999).

The year 2001 marks a second surge of solar activity, rivalling the activity peak one year before, at a point when the expected maximum of activity cycle 23 had already been passed. By chance, the year 2001 was also the first in which three stations of the R2S3 (Réseau de Suivi au Sol du Rayon Solaire) consortium carried out simultaneous continuous monitoring of the solar diameter, using CCD astrolabes. Since the three stations are geographically quite far apart, the conditions were the most favorable so far for comparing independent results from CCD solar astrolabes. Note that the solar astrolabe results constitute a lengthy, coherent set of solar diameter observations, covering two cycles of solar activity. However, long common patches of directly comparable observations are still lacking.

The principle of the determination of the solar diameter with CCD solar astrolabes is that of timing the crossing of the solar disk through an instrumentally defined zenith distance. The observation is automated, and the whole data reduction at the R2S3 sites follows common procedures. Details of the instrument (Delmas 2003a), of the observations (Jilinski et al. 1998), and of the data reduction (Sinceac 1998) have been fully given elsewhere. The effective wavelength of observation is basically defined by the shielding filter for the incoming rays and the sensitivity region of the CCD camera. It is about $5635 \AA$ for the three sites. The sites whose results are the subject of the present intercomparison are those 
of the Calern Station, Observatoire de la Côte d'Azur, France $\left(\phi=+43^{\circ} 45^{\prime} 02^{\prime \prime}, \lambda=-0^{\mathrm{h}} 27^{\mathrm{m}} 400^{\mathrm{s}} 0, h=1320 \mathrm{~m}\right)$, the Tubitak Observatory, Akdeniz Universitesi, Turkey $\left(\phi=+36^{\circ} 54^{\prime} 07^{\prime \prime}\right.$, $\left.\lambda=-2^{\mathrm{h}} 02^{\mathrm{m}} 37^{\mathrm{s}} \cdot 9, h=50 \mathrm{~m}\right)$, and the Observatório Nacional, Brasil ( $\left.\phi=-22^{\circ} 53^{\prime} 42^{\prime \prime}, \lambda=+2^{\mathrm{h}} 52^{\mathrm{m}} 53^{\mathrm{s}} .5, h=33 \mathrm{~m}\right)$. Specific details of these results have been presented by Delmas et al. (2003b), Kiliç et al. (2003) and Penna et al. (2003). It is worth mentioning here that the sufficiently long standing use of the method and algorithms, both at the same and at different stations, ensures that the features here examined and previously not present cannot be ascribed to defects of the program routines.

The next section presents the description of the series obtained at each site. In Sect. 3 the common stretches of the final series are statistically compared. In the final section the main conclusions are summarized and discussed.

\section{Results from each station}

The purpose of the R2S3 consortium is to provide homogeneous ground-based monitoring of solar diameter variations. Hence, the observations and data treatment are standardized. The results here presented employ only the standard outcomes from the R2S3 data treatment routines, plus a normalization, that is obtained by taking into account the variations of the Fried parameter. The Fried parameter is calculated from the dispersion of the projected trajectory of the extreme of the solar edge onto the focal plane. In this way, the results are normalized for variations of the instantaneous pixel scale caused by atmospheric turbulence, temperature and pressure variations, and the zenith distance of observation. The calculation of the Fried parameter is itself a standard by-product of the $\mathrm{R} 2 \mathrm{~S} 3$ data treatment routines.

Particular corrections introduced by each station at latter stages are not included here, for the sake of the direct comparison aimed at. Chief examples are air temperature corrections for the equatorial Rio de Janeiro station, and prism stability corrections for the higher-altitude Calern station observations. Notwithstanding their relevance when taking account of the detailed results from each station, the coefficients of the terms that were not included are all smaller than 0 ' 05 for normalized parameters. Their absence, therefore, does not affect the conclusions here obtained. Departures of the mean diameter value obtained at each station can be due to the different color of the frontal shielding filters; they are regarded as of no consequence for the present analysis which concerns the observed variations of the solar diameter.

\subsection{OCA/GEMINI - Calern station}

At the Calern station the observations are made using a front prism of variable angle (Delmas \& Laclare 2002). A rotating shutter alternates the CCD registration of the direct and reflected solar images. Observations are possible between $29^{\circ}$ and $61^{\circ}$ zenith distance, the lower angle corresponding to the interval from March to October. During 2001, bad weather prevented observations through most parts of April and May.
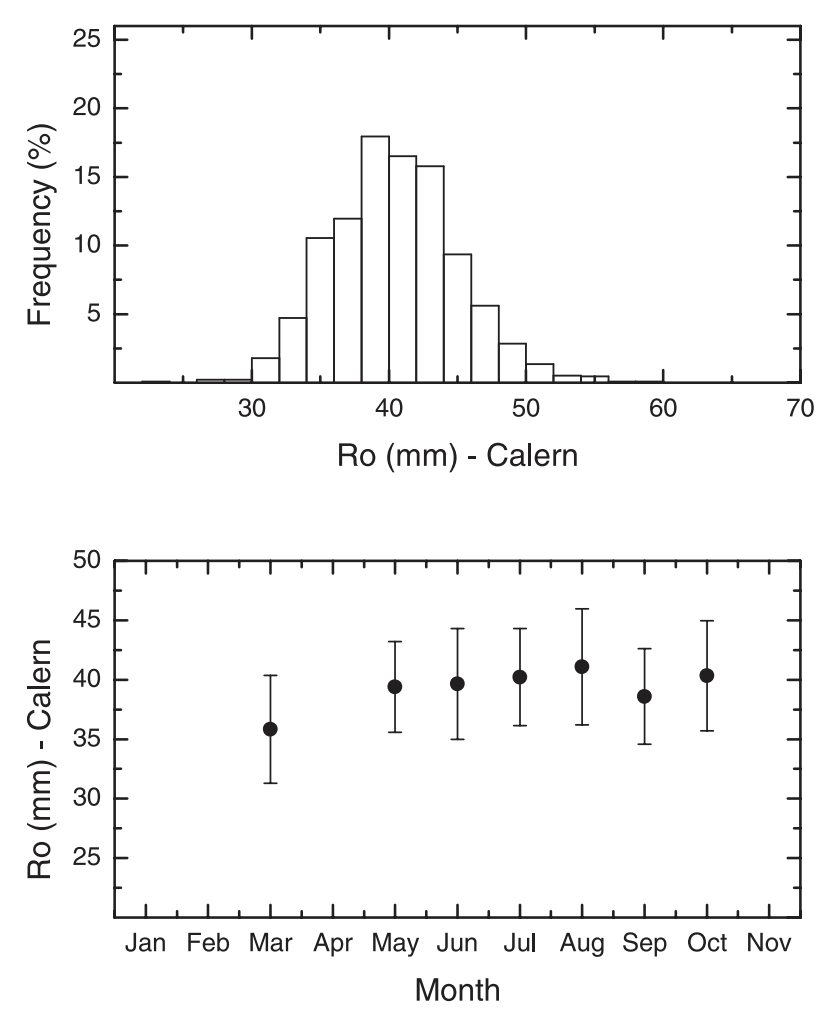

Fig. 1. Fried parameter frequency (Fig. 1a, top) and monthly average (Fig. 1b, bottom) distribution for the Calern series.

The distribution of the Fried parameter $\left(R_{0}\right)$, as obtained from the internal dispersion of each observation is shown in Figs. $1 \mathrm{a}$ and $1 \mathrm{~b}$. The average $R_{0}$ is $40.0 \mathrm{~mm}$, with standard deviation $4.5 \mathrm{~mm}$. Owing to the difference of the mean results, the Fried parameter normalization was calculated separately for the east and west observations. To take the Fried parameter into account, its reciprocal was taken $\left(1 / R_{0}\right)$. The reciprocals were normalized by subtracting the mean and multiplying by the standard deviation. In this way, after adjusting the observations, the obtained coefficient directly gives the average effect, in arc seconds. The coefficients were obtained to be $-0{ }^{\prime} 045 \pm 0{ }^{\prime} 020$ for the east observations, and $-0{ }^{\prime} 028 \pm 0{ }^{\prime} 020$ for west observations.

There is a total of 1318 observations, of which 754 are east and 564 west of the meridian. The final average semidiameter is 959!509 $\pm 00^{\prime} 014\left(\sigma=0{ }^{\prime} 490\right)$. The east minus west difference between the average semidiameters is $+0{ }^{\prime} 631 \pm 00^{\prime} 021$. Figures $2 \mathrm{a}$ and $2 \mathrm{~b}$ present the series distribution.

\subsection{Tubitak national observatory - Antalya station}

At the Antalya station the observations are made using sequentially three front prisms with angles corresponding to the zenith distances of $30^{\circ}, 45^{\circ}$, and $60^{\circ}$ (Golbasi et al. 2001). As at Calern, this enables observations from March to early November. The CCD registrations contain both the direct and reflected solar images. The distribution of the Fried parameter, as previously defined, is shown in Figs. $3 \mathrm{a}$ and $3 \mathrm{~b}$. The average $R_{0}$ is $47.0 \mathrm{~mm}$, with standard deviation $6.6 \mathrm{~mm}$. A single 

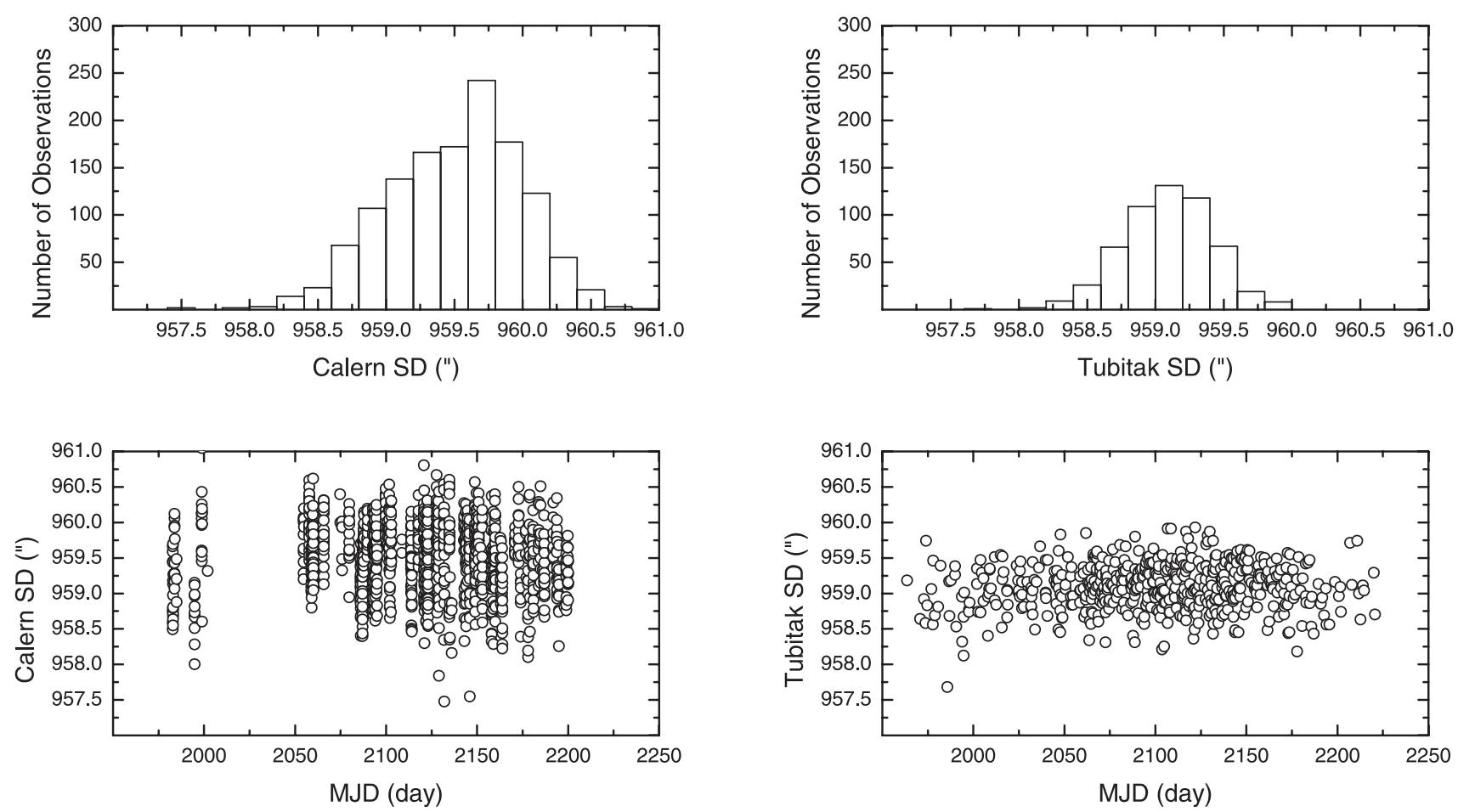

Fig. 2. Distribution of the residuals about the average (Fig. 2a, top) for the Calern semidiameter time series (Fig. 2b, bottom).
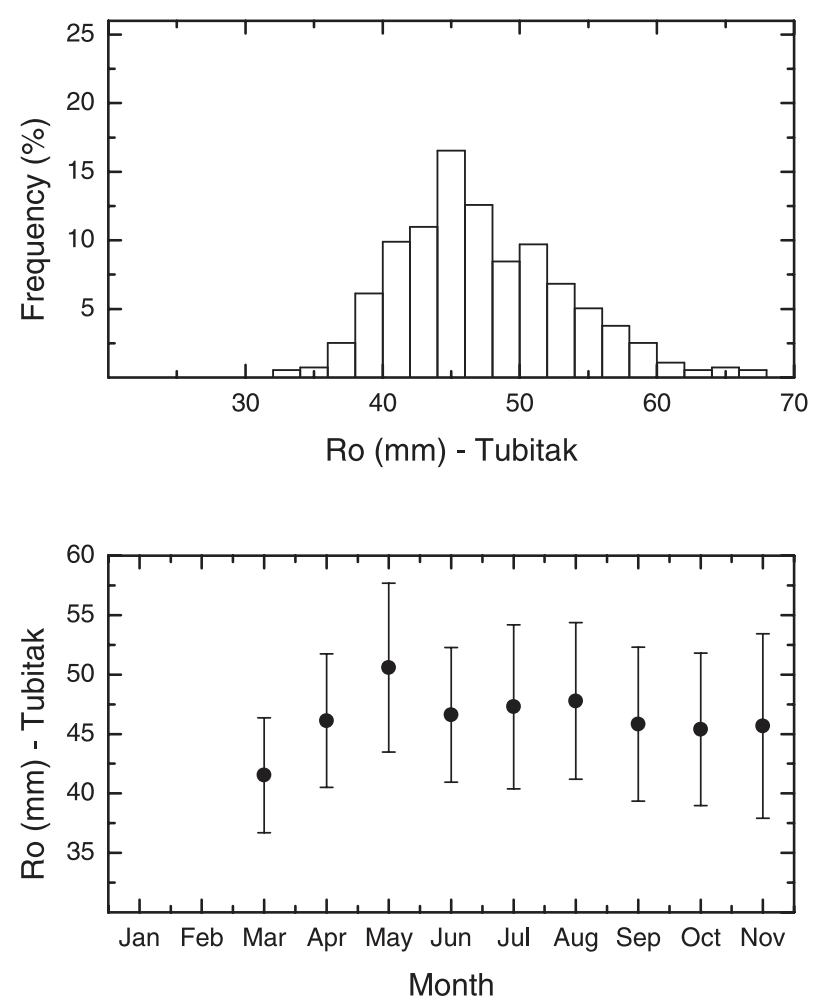

Fig. 3. Fried parameter frequency (Fig. 3a, top) and monthly average (Fig. 3b, bottom) distribution for the Tubitak series.

Fried parameter coefficient suffices for the eastern and western observations. The value of the coefficient is -0 ' $050 \pm$ $0 . ' 014$, as before obtained by adopting $1 / R_{0}$ as parameter and

Fig. 4. Distribution of the residuals about the average (Fig. 4a, top) for the Tubitak semidiameter time series (Fig. 4b, bottom).

normalizing. Again, the coefficient leads to a correction that does not alter substantially the measured values.

There is a total of 556 observations, 319 eastern and 237 western. The final average semidiameter is $959.091 \pm$ 0 '.014 ( $\sigma=0$ !'326). As implied above, the east minus west difference between the average semidiameters is $-0{ }^{\prime} 122 \pm 0 . ' 028$, much smaller than at the Calern station. Figures $4 \mathrm{a}$ and $4 \mathrm{~b}$ present the series distribution.

\subsection{ON - Rio de Janeiro station}

At the Rio de Janeiro station the two mentioned instrumental settings are combined (Jilinski et al. 1999). The observations are made using a front prism of variable angle, as at Calern, and the CCD registrations contain both the direct and reflected solar images, as at Antalya. The observations are possible between $26^{\circ}$ and $56^{\circ}$ zenith distance, all the year round (Penna et al. 2000). During 2001 however, extensive maintenance work on the front prism system and re-mirroring of the incoming rays shielding filter were done after September, which is then here adopted as the end of the series, to avoid unnecessarily introducing reconciliating factors. The distribution of the observational Fried parameter, as obtained from the internal dispersion of each observation is shown in Figs. 5a and $5 \mathrm{~b}$. The average $R_{0}$ is $36.7 \mathrm{~mm}$, with standard deviation $3.2 \mathrm{~mm}$. The coefficient of the normalized Fried parameter is $+0{ }^{\prime} \cdot 027 \pm 0 . ' 005$.

There is a total of 1890 observations, of which 976 are east and 914 are west of the meridian. A leveling correction, represented by an azimuthal dependence, was required. The final mean semidiameter is 959.'190 $\pm 0.013(\sigma=0 . ' 580)$. 

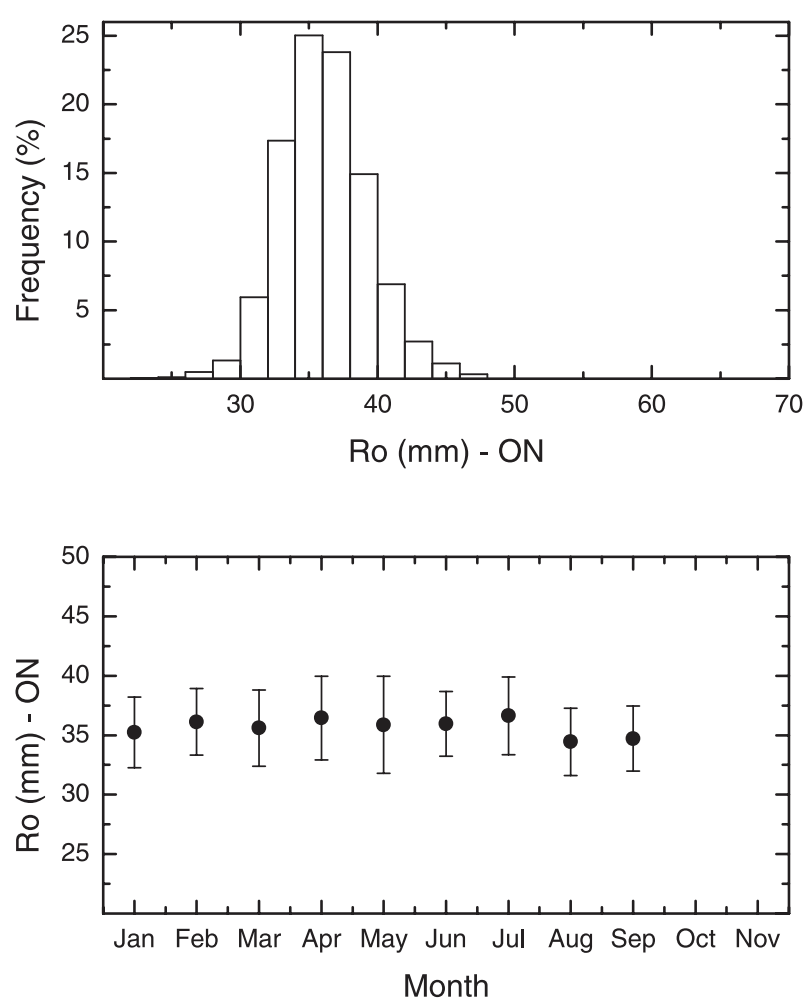

Fig. 5. Fried parameter frequency (Fig. 5a, top) and monthly average (Fig. 5b, bottom) distribution for the ON-Rio de Janeiro series.
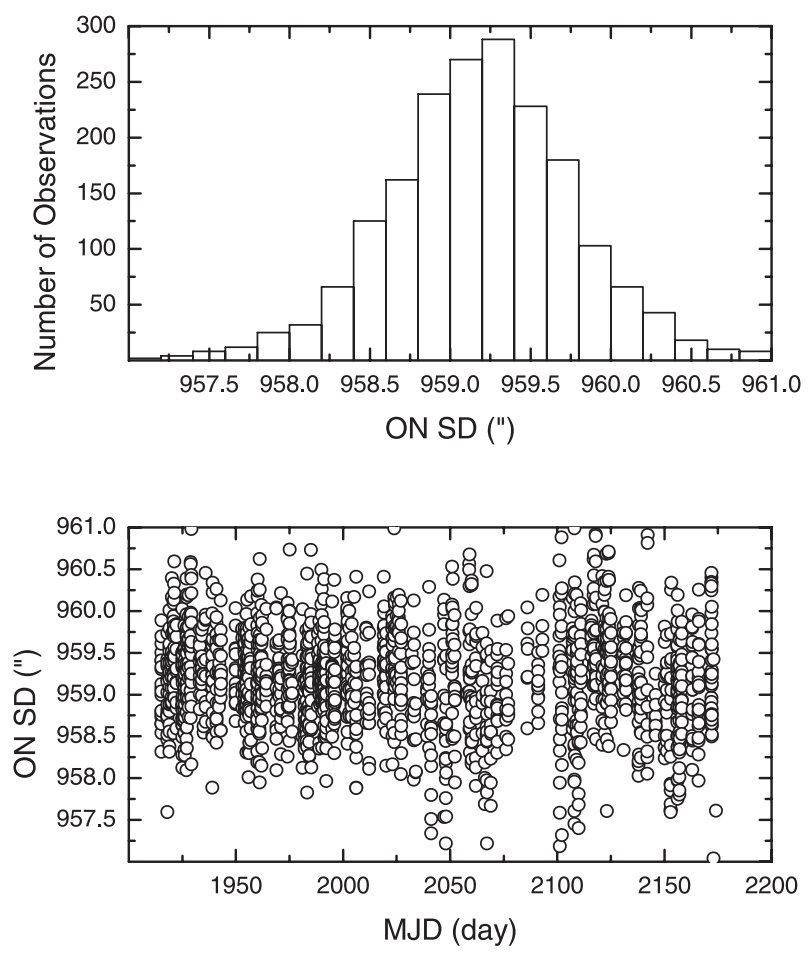

Fig. 6. Distribution of the residuals about the average (Fig. 6a, top) for the ON-Rio de Janeiro semidiameter time series (Fig. 6b, bottom).

The east minus west difference between the mean semidiameters is $-0 .{ }^{\prime} 016 \pm 0 .{ }^{\prime} 027$. Figures $6 \mathrm{a}$ and $6 \mathrm{~b}$ present the series distribution.

\section{Comparisons}

The inspection of the histograms in Figs. 2a, 4a, and 6a suggests that they do not exactly correspond to Normal distributions. The Anderson-Darling normality test $\mathrm{P}$ value is at the 0.001 level for the Calern and the ON-Rio de Janeiro series, and at 0.012 for the Tubitak series, not reaching the 0.05 critical $\mathrm{P}$ value of closeness to the normal distribution in any of the cases.

Next, the three series were tested for randomness by the Runs test. The null hypothesis (i.e., randomness) is not verified for any of the series. The significance is larger than 0.99 for the Calern and the ON-Rio de Janeiro series, and at 0.97 for the Tubitak series, therefore clearly above the 0.95 significance level.

Finally, all three time series exhibit non-zero autocorrelation. Seven significant lags are found for Calern, five for ON-Rio de Janeiro, and one for Tubitak. The difference in the number of significant lags reflects the time interval between successive observations, which is shortest for the fully automated astrolabe at Calern, and longest for the solar astrolabe of Tubitak with three fixed zenith distances

The combination of the three tests described above is interpreted as indicating that at least one significant trend can be found in the three semidiameter series, even though the signal to noise ratios are poor, as can be inferred from the standard deviations. This conclusion makes it possible to combine individual observations of the series to obtain smoothed series, in which trends and features can be detected above the noise.

Former studies indicate that, on time scales smaller than a year, the variations of the solar diameter are of the order of some hundredths of arc second (Andrei et al. 2001; Pap 2003). To get to this level of precision, loosely commensurable with fluctuations of the solar constant, starting from the accuracy of the individual measurements, the number of data points combined must be larger than one hundred. On the other hand, for the present investigation, both the peculiarities of the actual measurements and weakness of the expected short time scale variations of the solar diameter establish somewhere about a month as a safe lower limit for the bin size of time variations. During 2001 the number of observed days was 258 for Antalya, 219 for Calern, and 260 for ON-Rio de Janeiro. The total average, then, is 5.2 observations per day. From these considerations, the time series were smoothed by a FFT low pass band filter excluding frequencies higher than those corresponding to 150 points. Even for Antalya the filter is representative for the months that contain $62 \%$ of the measurements. The representative time series that will be used hereafter are shown in Fig. 7.

The representative time series depicted in Fig. 7 resemble each other both in the general trend and in the presence of a sizeable variation during the second half. Similar linear trends are obtained for the three series. For Tubitak the angular coefficient is $-5.4 \pm 0.4 \times 10^{-4}$, for Calern it is $-10.6 \pm 0.3 \times 10^{-4}$, and for ON-Rio de Janeiro it is $-6.3 \pm 0.4 \times 10^{-4}$, all in units of "/day. Clearly, the linear trend is only meant to be representative within the time interval studied here, as a common signature of the three series. It does not imply a secular trend, 


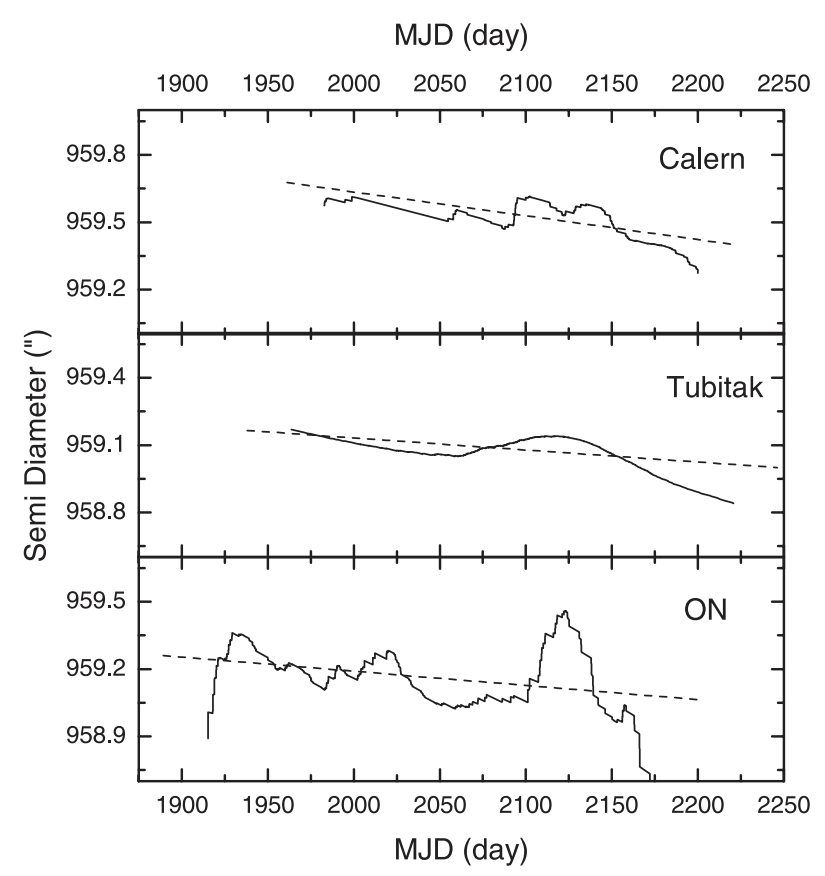

Fig. 7. Comparison of the three series of measured variations of solar semidiameter. The series are smoothed by a 150 points low pass band FFT. The dashed lines represent linear fits to the series. All horizontal axis follow the MJD scales given on the bottom and top parts of the figure. All vertical axes refer to semidiameter scales, in arc seconds.

Table 1. Pearson linear correlation coefficient for interpolations of the smoothed semidiameter variation series.

\begin{tabular}{lccc}
\hline \hline Sampling & \multicolumn{3}{c}{ Pairs } \\
\hline & Tubitak-Calern & Tubitak-ON & Calern-ON \\
\hline 180 points & 0.79 & 0.80 & 0.68 \\
120 points & 0.79 & 0.80 & 0.67 \\
60 points & 0.79 & 0.81 & 0.69 \\
30 points & 0.81 & 0.83 & 0.69 \\
20 points & 0.83 & 0.83 & 0.76 \\
10 points & 0.84 & 0.89 & 0.88 \\
\hline
\end{tabular}

or even a yearly trend. Long-time trends are not the subject here, and have been differently found by authors analyzing different time intervals (Laclare et al. 1996; Wittmann 2003; Noel 2004). Regarding the time interval studied here, however, it can be pointed out that Fig. 3 of Noel (2004) shows a peak feature that resembles the one found here.

To further compare the series, direct assessments of correlation were made. For this, the series were equally interpolated over the common period from MJD 1982 to 2174 . The interpolation goes from 180 points (i.e., close to 1 point/day) to 10 points (i.e., slightly above 1 point/month). Pearson linear correlations are presented in Table 1. As expected from the agreement of the linear regression results, the correlation is generally high, in particular for the pair Tubitak and ON-Rio de Janeiro. Although there is a progression it is modest, implying that the common linear trend is not the only matching characteristic.
Table 2. Significance level for the verified null hypothesis (at 95\%). between the smoothed semidiameter variation series.

\begin{tabular}{lccc}
\hline \hline Sampling & \multicolumn{3}{c}{ Pairs } \\
\hline & Tubitak-Calern & Tubitak-ON & Calern-ON \\
\hline 180 points & 0.90 & 0.78 & 0.31 \\
120 points & 0.93 & 0.88 & 0.41 \\
60 points & 0.96 & 0.96 & 0.69 \\
30 points & 0.96 & 0.94 & 0.73 \\
20 points & 0.95 & 0.90 & 0.74 \\
10 points & 0.97 & 0.85 & 0.97 \\
\hline
\end{tabular}

However, it is evident that the series are noisy, and besides, as previously discussed, the distributions depart from a Gaussian curve. Therefore, the nonparametric Mann-Whitney correlation test (which follows the Wilcoxon rank sum statistics) is better indicated to compare the distributions, regarding both the trend and the peak feature. The test was applied as two-sided, imposing the confidence level of 0.95 for accepting the null hypothesis. The null hypothesis is verified, that is, the pairs of normalized distributions behave statistically as random draws from the same population, for all pairs and all samples of interpolation. Table 2 gives the significance of the verified correlations. The significance grows rapidly when the analysis departs from the detailed 180 points interpolation samples. It nevertheless is always high for the pair Tubitak and Calern, which might be related to the similar regions of heliolatitude observed from the corresponding geographical latitudes. Yet the higher significance is to be be expected since this pair compares the smoothest series. The same reasons apply to the significance numbers for the Calern and ON-Rio de Janeiro verified correlation, namely lowest degree of smoothing and largest heliolatitude difference.

The peak feature locus agrees for the three representative series. It is located on MJD 2114.7 for Tubitak, on MJD 2123.1 for ON-Rio de Janeiro, and on MJD 2118.5 for Calern. Concerning the latter, it must be remarked that the local depletion in the region is actually due to an abnormal partitioning of the observations, once the average semidiameter for the west observations exceeds that from the east observations by $0 . ' 6$. The depletion becomes present because in the region the portion of west observations is $52.5 \%$, while for the whole series it is $40 \%$. Taking the east and west series separately, in both cases the depletion becomes the local maximum, surpassing by 0.03 the immediate left and right time neighborhoods. On the contrary, as measured from a baseline given by the adjusted regression lines, the height of the three peaks diverges in the case of the ON-Rio de Janeiro series. It is O' 05 for Tubitak, 0.' 1 for Calern, and 0.' 3 for ON-Rio de Janeiro. Again, it must be recalled that in the peak region the average observed heliolatitude was 55.1 ( $\sigma=16.7)$ for Rio de Janeiro, whereas it is $29^{\circ} .2\left(\sigma=19^{\circ} 8\right)$ for the Calern and $34^{\circ} .5\left(\sigma=14^{\circ} 2\right)$ for Tubitak. Therefore on the mid heliolatitude bulge of the solar figure (Rozelot et al. 2001) in the former case, and on the adjacent default in the later two. Whatever its cause, moreover, it is amplified by the fact that the ON-Rio de Janeiro representative series 


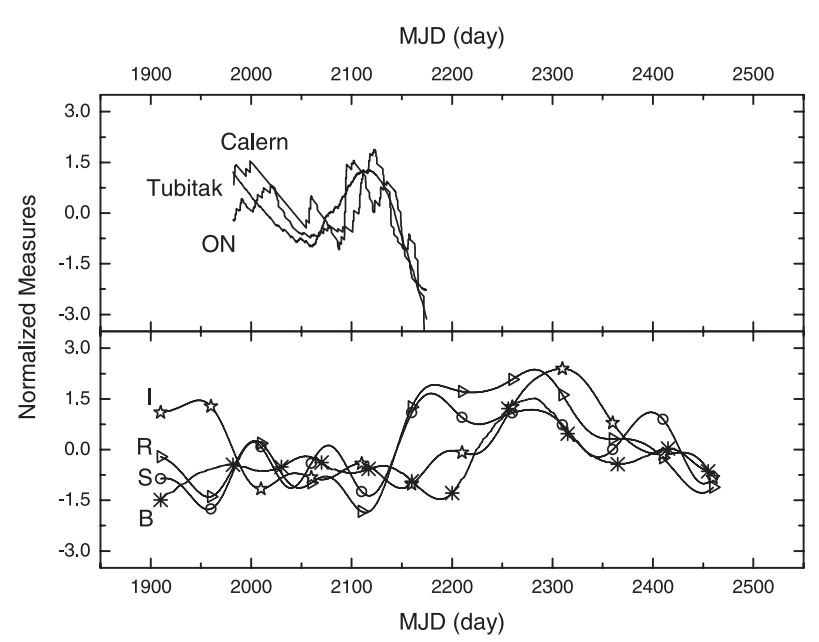

Fig. 8. Comparison of the normalized semidiameter variation time series (top) and the solar activity estimators series (bottom). In the bottom plot, I stands for the total solar irradiance (stars line), R stands for the $10.7 \mathrm{~cm}$ flux (triangles line), $\mathrm{S}$ stands for the sunspots number count (circles line), and B for the total strength of the solar magnetic field (asterisks line). All series are smoothed by a 150 points low passband FFT. All the series (semidiameter and solar activity estimators) are individually normalized, each by its mean and standard deviation.

is the least smoothed. If one adopts for the ON-Rio de Janeiro series an FFT smoothing by 380 points, equivalent to that of the Tubitak representative series, the peak height reduces to 0 .' 15 .

Figure 8 compares the three solar diameter times series and four estimators of the solar activity (see the acknowledgements section). The estimators are the $10.7 \mathrm{~cm}$ flux (line indicated by $\mathrm{R}$ and triangles), the sunspots count ( $\mathrm{S}$ and circles), the strength of the total solar magnetic field (B and asterisks), and the total solar irradiance (I and stars). In order to enable cross comparisons, both the semidiameter series and the estimators series have been individually normalized, each by its mean and standard deviation. Also for the sake of the comparison, the estimators series are smoothed by the same low pass band FFT, cutting out frequencies higher than those corresponding to one month. The semidiameter series are in agreement, but the estimators series are not. For these, the series appear to be shifted sidewards in time, as well as shifted relatively to the semidiameter series. Yet it can be noticed that the main peaks, both for the semidiameter and for the estimators series, are of similar heights. Within the limited scope allowed by the time interval of the figure, the scenario is of a surge in the solar diameter, the declining phase of which coincides with an increase in the sunspots number and in the $10.7 \mathrm{~cm}$ radio flux. During the second half of the increase in sunspots and radio flux, the strength of the total magnetic field rises and peaks. Finally, at the common decreasing of the sunspots count, of radio flux, and of the total magnetic field strength, the total solar irradiance reaches a maximum. Notice that the peak of the sunspot count coincides with a minimum of the total irradiance.

Such a scenario is in agreement with correlation tests. As before, the non parametric Mann-Whitney test was applied. To perform it, the estimator series were interpolated from 180 to 10 points, in a time interval equal to the length of that defined by the three semidiameter series. In the time domain of the semidiameter series, no correlation is found between any of the three semidiameter series against any of the four estimators of the solar activity. Actually, only for the 10 points interpolation, which reflects the broader tendencies, was a correlation verified of the semidiameter with the sunspot counts and the radio flux series, still at a low significance level (0.28). Equally, the correlation between the solar activity estimators is only verified at significance levels greater than 0.5 for the 10 points interpolation regime. All the correlations with significance larger than 0.5 involve the $10.7 \mathrm{~cm}$ radio flux. These are found, although at low significance, also for the 30 points and the 20 points interpolation regime.

This level of correlation improves considerably when the solar activity estimators series are phased by a time lag that shifts each of their main peaks toward the main peak of the semidiameter time series. The sunspot counts and the radio flux series are shifted by 54 days, the total magnetic field series by 150 days, and the total irradiance series is shifted by 180 days. As mentioned, in all cases the shift is toward the earlier dates. After the shift, the correlation between the semidiameter series and the sunspot count series, the radio flux series, and the total magnetic field is verified at an average significance level of 0.81 for the interpolations of 10,20 , and 30 points. Moreover, also for those interpolation sets, the correlations among the three estimators of the solar activity has average significance levels of 0.82 . Even for the most detailed interpolation sets $(180,120$, and 60 points) the average significance levels remain high, 0.68 for the correlations between the semidiameter and the solar activity estimators series; and 0.62 for the correlations among the solar activity estimators series.

Since the shifts given by the time distance between the semidiameter peak feature and the peaks of the different solar activity estimators are independent of the entire unfolding of any of the series, the large improvement in all the correlations supports the scenario above outlined. The higher levels of significance are obtained for the correlations involving the radio flux series, which presents the largest amplitude, coinciding with large portions of the peaks of all the other series. The total irradiance series only attains correlation with the other series for the 10 points interpolations, and at very low significance levels. This might indicate that to obtain a higher significance multi-correlation would be required for this estimator. It also states that, given the time length of the comparison, just adjusting the peaks so that they coincide would not yield the correlations obtained.

\section{Conclusions}

At Calern (France), Antalya (Turkey) and Rio de Janeiro (Brasil) the variations of the solar diameter are monitored with similar CCD solar astrolabes. The data acquisition and data reduction procedures are made following common standards of the Réseau de Suivi au Sol du Rayon Solaire. On the other hand, the atmospheric conditions at each station are completely different, as shown by the values derived for the Fried parameter. These conditions make it possible to assess the consistency of such measurements by a direct comparison of the results. 
A comparison was made for the time series of common measurements in the year 2001. Taking the three series, the average error for a single measurement is at 0.47 , but the number of measurements averages 5.2 observations per day. This enabled us to verify that background noise does not drown the signal, and that the signal is not compatible with a steady solar diameter throughout the period. The series were smoothed by low pass band FFT, with a 150 points step, that removes frequencies higher than those corresponding to a month.

The smoothed series revealed similar linear trends, averaging -0.7 mas/day, significant above $10 \sigma$. A large peak feature is verified for the three series, with the summit around MJD 2118. A conservative appraisal sets the height of the peak at $0{ }^{\prime} 1$ above the baseline given by the linear trends. That the series are in agreement is found by linear correlation, and by non-parametric verification of the null hypothesis.

The peak feature finds an apparent parallel to the important second maximum of solar activity appeared by the end of 2001 . Four estimators of the solar activity were compared, namely the $10.7 \mathrm{~cm}$ flux, the sunspot numbers, the strength of the total magnetic field, and the total irradiance. In the region covered by the comparison of the solar diameter variation series, significant correlation is obtained between the semidiameter variations and the solar activity estimators, as well as among these later, only when the different estimators peaks are shifted in time to match the peak of the variations of the semidiameter. The correlation cannot be due to the matching of the peaks, because complex features cover more than $70 \%$ of the various series. Accordingly, the most significant correlations involve the solar radio flux, while the total irradiance exhibit no simple, significant correlation.

Acknowledgements. The authors thank the support of several agencies: CNPq (Brazil), FAPERJ (Brazil)(E-26/150.496/2003), CNRS (France), and the Akdeniz University research fund. The authors acknowledge the use of Solar Irradiance data from PMOD/WRC, the
VIRGO Experiment, and the ESA/NASA Mission SoHO; the NOAA Daily Solar Data of radio flux and sunspot numbers; and the WSO Stanford Mean Solar Magnetic Field data.

\section{References}

Andrei, A. H., Penna, J. L., Reis Neto, E., et al. 2001, International Solar Cycle Studies 2001 - Solar Variability, Climate, and Space Weather, Longmont, USA, 13-16 June 2001

Chollet, F., \& Sinceac, V. 1999, A\&A, 139, 219

Delmas, C., \& Laclare, F. 2002, Sol. Phys., 209, 391

Delmas, C. 2003a, The Sun's Surface and Subsurface: Investigating Shape, ed. J.-P. Rozelot., Lect. Notes Phys., 599, 196

Delmas, C., Morand, F., \& Laclare, F. 2003b, Deuxìeme Réunion Annuelle R2S3, Calern, France, 24-26 November 2003

Emílio, M., Kuhn, J. R., Bush, R. I., \& Scherrer, P. 2000, ApJ, 543, 1007

Golbasi, O., Chollet, F., Kiliç, H., et al. 2001, A\&A, 368, 1077

Jilinski, E. G., Puliaev, S., Penna, J. L., et al. 1998, A\&AS, 130, 317

Jilinski, E. G., Puliaev, S., Penna, J. L., Andrei, A. H., \& Laclare, F. 1999, A\&AS, 135, 227

Kiliç, H., Golbasi, O., Chollet, F., \& Kiliç, D. 2003, Deuxìeme Réunion Annuelle R2S3, Calern, France, 24-26 November 2003

Laclare, F., Delmas, C., Coin, J. P., \& Irbah, A. 1996, Sol. Phys., 166, 211

Lakhal, L., Irbah, A., Bouzaria, M., et al. 1999, A\&AS, 138, 155

Noel, F. 2004, A\&A, 413, 725

Pap, J. M. 2003, The Sun's Surface and Subsurface: Investigating Shape, ed. J.-P. Rozelot, Lect. Notes Phys., 599, 129

Penna, J. L., Jilinski, E., Andrei, A. H., Puliaev, S., \& Reis Neto, E. 2002, A\&AS, 384, 650

Penna, J. L., Jilinski, E. G., Andrei, A. H., et al. 2003, Deuxìeme Réunion Annuelle R2S3, Calern, France, 24-26 November 2003

Reis Neto, E., Andrei, A. H., Penna, J. L., Jilinski, E. G., \& Puliaev, S. P. 2003, Sol. Phys., 212, 7

Rozelot, J. P., Godier, S., \& Lefebvre, S. 2001, Sol. Phys., 198, 223

Sinceac, V. 1998, Ph.D. Thesis, Paris Observatory

Wittmann, A. D. 1997, Sol. Phys. 171, 231

Wittmann, A. D., 2003, Astron. Nachr., 324, 378 